\title{
Managing the Office
}

\section{The Elements}

1 The Role of the Office

2 Communication and the Office

3 Information Handling in Office Services $10-11 \mathrm{hrs}$

4 The Design of Office Systems

5 The Introduction and Evaluation of Office Systems

6 Financial Systems within the Office

7 Personnel Administration within the Office

8 The Supervision of Office Staff

9 Organising and Running Meetings

\section{Guide} Time Elements *

8-10 hrs

9-10 hrs

1

1

$1,2,3$

12-15 hrs

8-9 hrs

$1,2,3,4$

12-13 hrs

$1,2,3,4,5$

9-10 hrs

1,2

10-11 hrs

1,2

8-9 hrs

1,2

* This column gives the reference numbers of any other Elements of this Unit which contain necessary prior knowledge. Before starting this Element you should either have completed these prior Element(s), or have gained the relevant knowledge in some other way. 